\title{
Piecewise Potential Vorticity Inversion without Far-Field Response?
}

\author{
JOSEPH EGGER ${ }^{\mathrm{a}}$ AND Klaus P. HOINKA ${ }^{\mathrm{b}}$

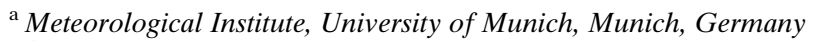 \\ ${ }^{\mathrm{b}}$ Institute of Atmospheric Physics, Oberpfaffenhofen, Germany
}

(Manuscript received 13 May 2020, in final form 27 August 2020)

\begin{abstract}
Given a flow domain D with subdomains D1 and D2, piecewise potential vorticity inversion (PPVI) inverts a potential vorticity (PV) anomaly in D2 and assumes vanishing PV in D1 where boundary conditions must be taken into account. It is a widely held view that the PV anomaly exerts a far-field influence on D1, which is revealed by PPVI. Tests of this assertion are conducted using a simple quasigeostrophic model where an upper layer D2 contains a PV anomaly and D1 is the layer underneath. This anomaly is inverted. Any downward physical impact of PV in D2 must also be represented in the results of a downward piecewise density inversion (PDI) based on the hydrostatic relation and the density in D2 as following from PPVI. There is no doubt about the impact of the mass in D2 on the flow in the lower layer D1. Thus results of PPVI and PDI have to agree closely. First, PPVI is applied to a locally confined PV anomaly in D2. There is no far-field "response" in D1 if stationarity is imposed. Modifications of boundary conditions lead to "induced" flows in D1 but the results of PPVI and PDI differ widely. This leads to a simple proof that there is no physical far-field influence of PV anomalies in D2. Wave patterns of the streamfunction restricted to D2 are prescribed in a second series of tests. The related PV anomalies are obtained by differentiation and are also confined to D2 in this case. This approach illustrates the basic procedure to derive PV fields from observations which excludes a far-field response.
\end{abstract}

KEYWORD: Atmospheric circulation

\section{Introduction}

It is one of the main results of quasigeostrophic theory that potential vorticity (PV) can be predicted using only the PV conservation equation. Complete information on the PV field at some time is sufficient to forecast PV and the related flow provided the boundary conditions can be satisfied. This "PV-perspective" (Hoskins et al. 2003) has been adopted many times in the past (e.g., Phillips 1954; Bleck and Mattocks 1984) and is described in most textbooks on dynamic meteorology. A plausible and meteorologically relevant illustration of these techniques has been proposed by Hoskins et al. (1985, henceforth HMR) in a thought experiment where a positive upper-level PV anomaly in quiescent air is specified above a lower layer with an airstream of mean zonal velocity $U$ but with $\mathrm{PV}=0$ (Fig. 1). Inversion of this PV field is expected to generate a cyclonic flow feature V1 underneath the stationary anomaly. HMR suggest "that the cyclonic vortex in the lower troposphere must stay in place."

This is an example of piecewise PV inversion (PPVI) where a PV anomaly is selected from a flow field in order to find the "response" of the flow to the anomaly. In general there are more than one anomaly in published cases. The most promising ones are then selected for PPVI. However, the inversion problem with respect to every one is essentially the same as that posed by Fig. 1 so that we may stay with the simple case in Fig. 1 where questions concerning the impact of PV on the flow can be addressed. By and large, there appear two groups of interpretations of PPVI in the literature. It is the leading view that any "localized PV anomaly contributes to the flow in the far-field" and that there is an "action at a distance effect" (Davies and Bishop 1994). HMR suggested that PV

Corresponding author: Joseph Egger, j.egger@lrz.uni-muenchen.de anomalies "induce" circulations and that the induced fields penetrate vertically above and below the anomalies. Bishop and Thorpe (1994) attributed to PV anomalies an unique influence on the rest of the atmosphere in analogy to the situation in electrostatics. Bretherton (1966) pointed out that even the boundary values of temperature can be seen as singular PV anomalies in infinitely thin sheets. These anomalies are then thought to affect the flow at a distance. A careful discussion of these issues may be found in Thorpe (1997). Such a far-field influence is not claimed in simpler interpretations which see PPVI as a mathematical problem (Hakim et al. 1996; Egger 2008) where one looks for that flow in D = D1 + D2, which forms, for example, the PV field of Fig. 1 with the specified anomaly in D2 and vanishing PV in D1. It is an important point in PPVI as stressed, for example, by Hakim et al. (1996) that the boundary conditions have a large influence on the results of PPVI. This influence must be taken into account when discussing an eventual impact of PV anomalies.

A clarification of these important issues concerning the interpretation of PPVI is intended here by conducting tests in a quasigeostrophic two layer model. The layer above the height $z=0$ in Fig. 1 serves as a source region D2 and the layer D1 is the target region. PPVI for such a configuration has been performed quite often (e.g., Robinson 1988; Hartley et al. 1998; Baumgart et al. 2018). Inversion of the rectangular PV field in Fig. 1 is relatively simple in the two dimensional quasigeostrophic frame where PV is

$$
q=\frac{\partial^{2} \psi}{\partial x^{2}}+\frac{\partial^{2} \psi}{\partial z^{2}}
$$

with streamfunction $\psi$. It is assumed in (1) for the sake of simplicity that the mean density $\bar{\rho}$ is constant as are the BruntVäisälä frequency $N_{B}$ and the Coriolis parameter $f$. The 


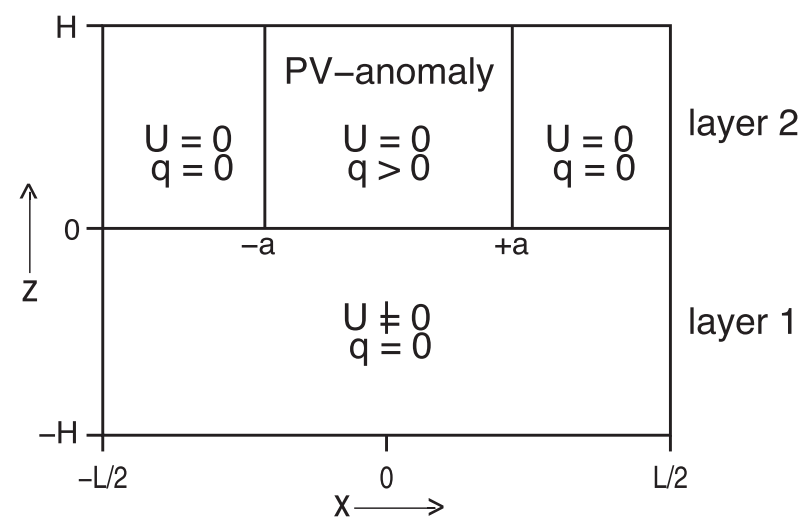

FIG. 1. Schematic of the flow considered in the thought experiment of HMR. The PV anomaly $q>0$ is restricted to the domain $-a \leq x \leq a, 0 \leq z \leq H$. Zonal mean flow is denoted by $U$. Continuity of $\psi$ and $w$ at $z=0$.

vertical coordinate $z$ in (1) is stretched by the factor $N_{B} / f$ with respect to the standard height coordinate. Given $q$, (1) has to be solved taking the boundary conditions into account. The scenario proposed by HMR appears to be ideal for such investigations of eventual far-field influence because the PV anomaly in D2 is stationary. A further wave-shaped PV anomaly will be considered as well.

The issue of an eventual physical influence of PV anomalies can be resolved by performing inversions of density in parallel to those of PV. Assume that a flow perturbation $\psi^{\prime}$ results by inversion of a PV anomaly in $\mathrm{D} 2$. A density deviation $\rho^{\prime}=-\left(N_{B} \bar{\rho} / g\right)\left(\partial \psi^{\prime} / \partial z\right)$ is associated with this inverted flow. The hydrostatic approximation can then be integrated downward from the top level $z=H$ to yield

$$
\psi_{d}^{\prime}(z)=\frac{1}{\bar{\rho} N_{B}} \int_{z}^{H} g \rho^{\prime} d z^{\prime \prime}+\psi_{d}^{\prime}(H),
$$

where the subscript $d$ refers to density inversion and $\psi_{d}^{\prime}(H)$ is the prescribed perturbation streamfunction on top which is related to the pressure perturbation $p_{d}^{\prime}$ by $\psi_{d}^{\prime}=p_{d}^{\prime} /(\bar{\rho} f)$. The interpretation of (2) is obvious. Gravity attracts the mass in the layer $z \leq z^{\prime \prime} \leq H$. This force is balanced by the pressure gradient force and $\psi_{d}^{\prime}(z)$ represents the impact of this mass. If, in particular, $\psi_{d}^{\prime}(H)=0$ the impact of layer D2 on D1 is given by $\psi_{d}^{\prime}(0)$ which is felt at all levels of height $z<0$. This is an undisputed far-field effect which acts, however, in vertical direction only. This influence does not depend on height in D1. Thus any physical influence of PV in D2 on the flow below must be quite similar to that revealed by density inversion. Of course, the results of PPVI and PDI are the same in D2. Piecewise inversion of potential temperature has been related to PPVI by Egger and Hoinka (2010), who did not discuss, however, the physical impact of anomalies.

\section{The model}

A zonally periodic atmosphere in a two-dimensional domain of length $L$ and depth $2 H$ is chosen to deal with the interpretation of PPVI (see Fig. 1). Vanishing PV is prescribed in D except for an anomaly to be specified in $\mathrm{D} 2$. The mean flow is $U=0$ in the upper layer D2 $(0 \leq z \leq H)$ and $U=U_{z} z$ with constant shear $U_{z}$, in the lower layer D1 $(-H \leq z \leq 0)$ so that the mean flow $\mathrm{PV}$ vanishes in both layers. Thus $\psi=-U y+\psi^{\prime}$ with perturbation streamfunction $\psi^{\prime}$. The flow equations are linearized with respect to this mean flow. They are needed here only in the discussion of stationarity. Altogether, this is simply an Eady model with a "stratosphere." It is a two-layer model with matching conditions at $z=0$. PV can be evaluated in each layer but is not defined at $z=0$. A similar but more general model with vanishing PV has been analyzed by Hoskins et al. (2003). The simpler model chosen here is, however, sufficiently complex for our purposes.

Two types of PV anomalies will be considered. The anomaly $Q_{L}$ is localized as in Fig. 1. A uniform PV distribution with $q=$ $q_{0}$ is prescribed in the rectangular domain $0 \leq z \leq H,-a \leq z \leq$ a. A wavelike anomaly $Q_{W}=\tilde{q}(z) \sin (2 \pi x / L)$ is inserted in D2 in a further series of inversions. The profile $\tilde{q}(z)$ will be specified later.

The thermodynamic energy equation is

$$
\left(\frac{\partial}{\partial t}+U \frac{\partial}{\partial x}\right) \frac{\partial \psi^{\prime}}{\partial z}-\frac{\partial \psi^{\prime}}{\partial x} U_{z}+f w=,
$$

with $U_{z}=0$ in D2 and vertical velocity $w$. The layers D1 and D2 join at $z=0$ and there are rigid lids at $\pm H$. Continuity of $\psi$ at $z=0$ is a necessary condition but continuity of "temperature" $\partial \psi^{\prime} / \partial z$ is desirable in addition. The conditions

$$
\left(\frac{\partial}{\partial t}-U_{z} H \frac{\partial}{\partial x}\right) \frac{\partial \psi^{\prime}}{\partial z}-U_{z} \frac{\partial \psi^{\prime}}{\partial x}=0
$$

at $z=-H$ and

$$
\frac{\partial^{2} \psi^{\prime}}{\partial t \partial z}=0
$$

at $z=H$ follow from (3) because $w=0$ at the horizontal boundaries. Analytic solutions of (1) for $Q_{L}$ will be presented next.

\section{Results of PPVI}

a. Anomaly $Q_{L}$

It is convenient to conduct the analysis in Fourier space with Fourier transform $\hat{\psi}_{n}\left(k_{n}, z\right)$ of $\psi$ with zonal wavenumber $k_{n}=$ $2 \pi n / L(1 \leq n \leq N)$. The transform of $q$ is related to that $\psi$ of by

$$
\hat{q}_{n}=\left(-k_{n}^{2}+\frac{\partial^{2}}{\partial z^{2}}\right) \hat{\psi}_{n} .
$$

The solution of (1) for $Q_{L}$ can be based on the Fourier expansion

$$
q^{\prime}=\sum_{n=1}^{N} \hat{q}_{2 n} \cos \left(k_{n} x\right)
$$

for $z>0, \hat{q}_{2 n}=2 q_{0} \sin \left(k_{n} a\right) /(n \pi)$ but $\hat{q}_{1 n}=0$ for $z<0$. The related modes of $\psi^{\prime}$ are 


$$
\hat{\psi}_{2 n 0}=-\hat{q}_{2 n} / k_{n}^{2}
$$

because $\hat{q}_{2 n}$ does not depend on $z$. Thus $\hat{\psi}_{2 n 0}$ is the result of PVI in D2 for wavenumber $n$. Of course, $\hat{\psi}_{1 n 0}=0$ in D1. The subscript 1 (2) denotes the lower (upper) layer. Further modes $\sim \exp \left( \pm k_{n} z\right)$ of vanishing PV must be added to satisfy boundary and interface conditions.

Boundary conditions are somewhat arbitrary in PPVI. It is impossible to ascribe to a PV anomaly a unique set of boundary conditions. Observed boundary values are not of help in realistic cases of PPVI because they cannot be associated with a single anomaly. It is customary to prescribe vanishing temperatures at the horizontal boundaries in keeping with the idea of Bretherton (1966). On the other hand this choice implies in general a nonvanishing profile of $\psi$ at the upper boundary. One has to ascribe the related pressure to masses above $z=H$ but it is difficult to discuss the physical impact of PV if part of the related flow is unrelated to PV. Thus it is an attractive option to prescribe $\psi=0$ at $z=H$ and $\partial \psi / \partial z=0$ at $z=-H$.

The discussion of the problem could have been simplified by choosing a sinusoidal distribution of $q$ in D2 so that only one wavenumber would have to be included. However, the connection to the intriguing situation proposed by HMR would have been lost this way. An anomaly with a single wave will be considered below.

As stressed by Hakim et al. (1996) the inverted streamfunction should also represent the area integral $\left(2 a H q_{0}\right)$ of $Q_{L}$ but the Fourier representation (7) implies a vanishing mean value. There are several ways to solve this problem but the simplest is presumably to insert another PV anomaly $Q_{L}^{*}$ in D2 which has the same shape as $Q_{L}$ but with uniform $\left(q=-q_{0}\right)$. This additional anomaly has the same impact on D1 as $Q_{L}$ except for zonal location and sign. It simplifies, however, the discussion to restrict the presentation of the results to those related to $Q_{L}$.

Following HMR we expect that there is a stationary solution of the model equations. Stationarity implies $w=0$ in D2 [see (3)] and at $z=0$ so that

$$
\frac{\partial}{\partial t}\left(\frac{\partial \psi^{\prime}}{\partial z}\right)-U_{z} \frac{\partial \psi^{\prime}}{\partial x}=0
$$

at $z=0$ in D1. Stationarity is possible only for $\psi^{\prime}=0$ at $z=0$. This simple result implies $\psi^{\prime}=0$ in D1 at least if the lower boundary condition requires $\psi^{\prime}$ or $\left(\partial \psi^{\prime} / \partial z=0\right)$ to vanish at $z=-H$. These conditions can be satisfied by adding properly chosen modes with vanishing PV. Simple calculations lead to the stationary result of the inversion of $Q_{L}$ in Fourier components

$$
\hat{\psi}_{2 n s}=\hat{\psi}_{2 n 0}\left[1-\gamma_{n} \exp \left(k_{n} z\right)-\left(1-\gamma_{n}\right) \exp \left(-k_{n} z\right)\right],
$$

with $\gamma_{n}=\left[1-\exp \left(-k_{n} H\right)\right] /\left[2 \sinh \left(k_{n} H\right)\right]$ and the subscript $s$ for stationarity. Indeed $\psi_{s}^{\prime}=0$ at $z=0$ and the perturbation streamfunction vanishes at $z=H$. Of course, $\psi_{s}^{\prime}=0$ in D1. This solution of $\psi^{\prime}$ is shown in Fig. 2. The pressure minimum is located near $z=H / 2, x=0$ with positive values of $\psi^{\prime}$ near the lateral boundaries. There is no cyclonic flow underneath $Q_{L}$. The PV anomaly $Q_{L}$ does not exert a downward influence. This

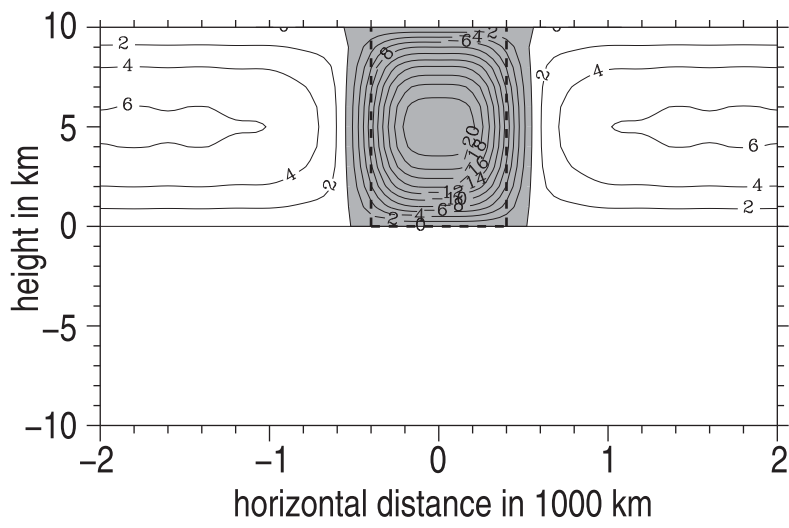

FIG. 2. Streamfunction perturbation $\psi^{\prime}$ (isolines: $2.0 \times 10^{4} \mathrm{~m}^{2} \mathrm{~s}^{-1}$ ) resulting from the inversion (10) of the PV anomaly $q_{0}=10^{-5} \mathrm{~s}^{-1}$ prescribed in the rectangular domain with dashed boundaries; negative values shaded. $B_{N}=5.0 \times 10^{-3} \mathrm{~s}^{-1}, U_{z}=10^{-5} \mathrm{~s}^{-1}, a=$ $4.0 \times 10^{5} \mathrm{~m} ; N=10$.

is a somewhat surprising result in view of the standard interpretation of PPVI. Note that $\partial \psi / \partial z$ is not continuous at $z=0$. This is compatible with the structure of the model [see also Hoskins et al. (2003) and further comments below].

Density inversion must be based on the "temperature" $\partial \psi_{s}^{\prime} / \partial z$ provided by PPVI in D2. It is sufficient to look at $\psi_{2 s}^{\prime}(0)$ and $\psi_{2 s}^{\prime}(H)$. The streamfunction vanishes at $z=H$ according to (10) so that the impact of the mass in D2 on the flow in D1 is given by $\psi_{1 s}^{\prime}(z)=\psi_{1 s}^{\prime}(0)=0$. Thus there is no far-field influence.

As pointed out by a reviewer one may see in Fig. 2 a sheet of negatively signed boundary PV at $z=0$ which "shields" the influence of the positive PV in the upper layer on the lower layer. Note that this singular anomaly of PV at $z=0$ is generated by the inversion. In principle, full information on the PV field must be available before inversion. It is straightforward to invert the boundary PV. The result is $\psi_{b n}=\hat{\psi}_{2 n}\left(1-2 \gamma_{n}\right) \sinh [k(z-H)] / \cosh (k H)$ for $0 \leq z \leq H$ with $\psi_{b n}(H)=0$ and the correct value of $\partial \psi_{b n} / \partial z$ at $z=0$. It is not clear in which sense $\psi_{b}$ contributes to (10) or shields D1 (see also Egger 2008).

The solution (10) is stationary but $\partial \psi / \partial z$ is not continuous. A solution which satisfies continuity of $\psi^{\prime}$ and $\partial \psi^{\prime} / \partial z$ at $z=0$ must be of the form

$$
\begin{aligned}
& \hat{\psi}_{2}=\hat{\psi}_{20}\{1-\cosh [k(z-H)]+A \sinh [k(z-H)]\}, \\
& \hat{\psi}_{1}=B \cosh [k(z+H)],
\end{aligned}
$$

so that $\hat{\psi}_{2}=0$ at $z=H$ and $\partial \psi_{1} / \partial z=0$ at $z=-H$. The subscript $n$ is omitted. The coefficients $A$ and $B$ follow after imposing both conditions. The result is displayed in Fig. 3a.

There is cyclonic flow and low pressure in the domain of $Q_{L}$. This region extends down to the bottom and may be identified with the vortex V1 mentioned above. This pattern suggests seeing here a far-field influence of $Q_{L}$ but the density inversion refutes this claim. The far-field influence of the layer D2 on $\mathrm{D} 1$ is described by $\psi^{\prime}(0)$ (see Fig. 3a). The mass in D2 contributes low pressure underneath the anomaly and high pressure 


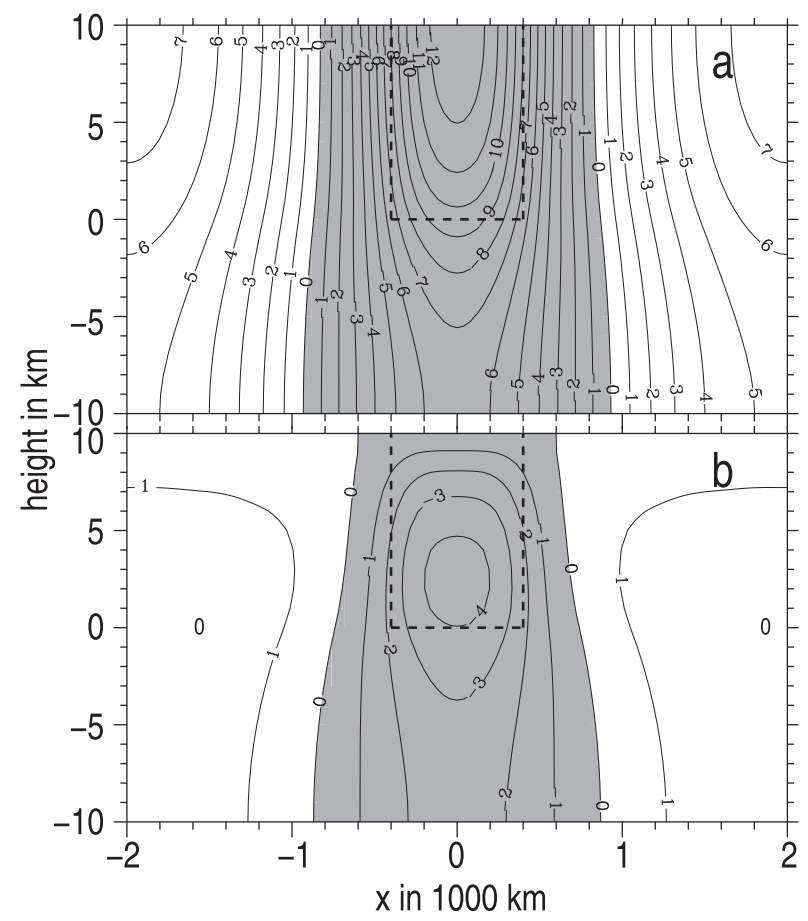

FIG. 3. Streamfunction $\psi$ in $10^{5} \mathrm{~m}^{2} \mathrm{~s}^{-1}$ : (a) $\partial \psi / \partial z=0$ at $z \pm H$ and (b) $\psi=0$ at $z=H, \partial \psi / \partial z=0$ at $z=H ; N=10$.

further off. This impact does not depend on height. The potential vorticity of the PDI flow in D1 equals the vorticity $\partial^{2} \psi^{\prime} / \partial x^{2}=0$ of $\psi^{\prime}(0)$. The mass in D2 induces a PV field in D1 while PPVI requires PV $=0$ in D1 as in Fig. 3a. The inverted flow in D1 in Fig. 3a cannot be interpreted as a physical far-field influence of $Q_{L}$.

The result of PPVI is not much different if vanishing temperatures are assumed on top (Fig. 3b). The cyclonic anomaly is weaker in Fig. 3a. The pressure at $z=H$ has an impact on the flow in D1, of course. Again, PDI produces a height independent contribution in D1 with PV anomalies. It is now easy to demonstrate that a physical impact of a PV anomaly in D2 on the flow in D1 is impossible. This impact would have to be described by combination of functions $\sim \exp$ $[-k(2+H)], \exp [k(2+H)]$ as in (11). The specific form of this combination depends on the boundary conditions but there is no doubt that this combined function depends on height, that is, it cannot express the physical impact which does not depend on $z$. Of course there is no height dependence if this function vanishes as in (10) but there is then no impact anyway.

Dr. Heifetz suggested to revert Fig. 3 almost to Fig. 2 by adding a singularity of PV at $z=0$ in the sense of Bretherton (1966) such that the Green function of this imposed jump of $\partial \psi^{\prime} / \partial z$ at $z=0$ cancels the streamfunction for $z<0$ as given in Fig. 3. The agreement with Fig. 3 is not perfect because $\psi_{2}^{\prime}(H) \neq 0$ in this case. The sum of $Q_{L}$ and the new singular PV provides thus a further example of vanishing flow underneath a PV anomaly in D2. This time the concept of "shielding" is well illustrated.
One may argue that the flow in Fig. 3a is superior to that in Fig. 2 because the temperature at $z=0$ is continuous in Fig. 3a. However, this continuity is singular in the sense that it is not maintained in time. A discontinuity will be established immediately as follows from (3) with continuity of $w$ :

$$
\frac{\partial}{\partial t}\left(\frac{\partial \psi_{2}^{\prime}}{\partial z}-\frac{\psi_{1}^{\prime}}{\partial z}\right)=U_{z} \frac{\partial \psi_{1}^{\prime}}{\partial x} \neq 0
$$

The temperature difference will grow in time. Thus jumps of temperature at $z=0$ are inherent in the model.

The flows in Fig. 3 can be chosen as initial states in integrations of the PV equation

$$
\left(\frac{\partial}{\partial t}+U \frac{\partial}{\partial x}\right) q=0,
$$

where (4) and (5) have to be imposed at the horizontal boundaries. The resulting flows will not be presented. They are the sum of the stationary solution (10) and components with vanishing PV which satisfy the initial and boundary conditions. An eigenvalue evaluation has been performed for this mean flow profile. As one would expect, long waves are unstable. Thus we obtain in time an infinitely large group of flows associated with the stationary PV anomalies.

\section{b. Anomaly $Q_{W}$}

The approach to the issue of far-field influence chosen here is simpler than that in the foregoing section. A suitable streamfunction $\psi_{w}^{\prime}$ is prescribed and $\mathrm{PV}$ is derived from that. Let us prescribe an "observed" streamfunction

$$
\psi_{w}^{\prime}=P z^{3} \sin \left(k_{1} x\right)
$$

for $z>0$ with amplitude factor $P$ and $\psi_{w}^{\prime}=0$ for $z \leq 0$. This streamfunction is antisymmetric with respect to the origin. The related $\mathrm{PV}$ anomaly is

$$
Q_{W}=\left(6 P z-k_{1}^{2} P z^{3}\right) \sin \left(k_{1} x\right)
$$

for $z>0$ and $Q_{W}=0$ for $z<0$. The mean value of $Q_{W}$ vanishes unlike that of $Q_{W}$. The streamfunction $\psi_{w}^{\prime}$ is defined such that $\psi_{w}^{\prime}$ and the temperature $\partial \psi_{w}^{\prime} / \partial z$ vanish at $z=0$. Thus (14) is analogous to (10) but $\psi_{s}$ is obtained by PPVI while $\psi_{w}$ is simply prescribed. It would make little sense to ascribe to $Q_{W}$ a downward impact because $Q_{W}$ is simply derived from $\psi_{w}^{\prime}$ as is standard in quasigeostrophy. PDI is not needed in this case.

Both $\psi_{w}^{\prime}$ and $Q_{W}$ are displayed in Fig. 4 with extrema at $z=$ $H$ and vanishing values at for $z<0$. The first term in the parentheses in (15) dominates for $z<6 / k_{1} \sim 1.5 \times 10^{5} \mathrm{~m}$ for the parameters in Fig. 4. Thus $Q_{W}>0$ for all $z>0, x>0$ due to the upward increase of temperature. The factor $P$ in (14) is chosen such that $Q_{W}=10^{-4} \sin \left(k_{1} x\right) \mathrm{s}^{-1}$ on top at $x=L / 2$.

A more elegant version of this approach can be implemented by prescribing

$$
\psi_{w}^{*}=P z^{2}(z-H) \sin \left(k_{1} x\right)
$$

for $z>0$ and $\psi_{w}^{*}=0$ for $z<0$. Thus $\psi_{w}^{*}=0$ for $z=H$ and (16) satisfies all conditions for a streamfunction which can be interpreted as the result of PPVI. The corresponding PV is 


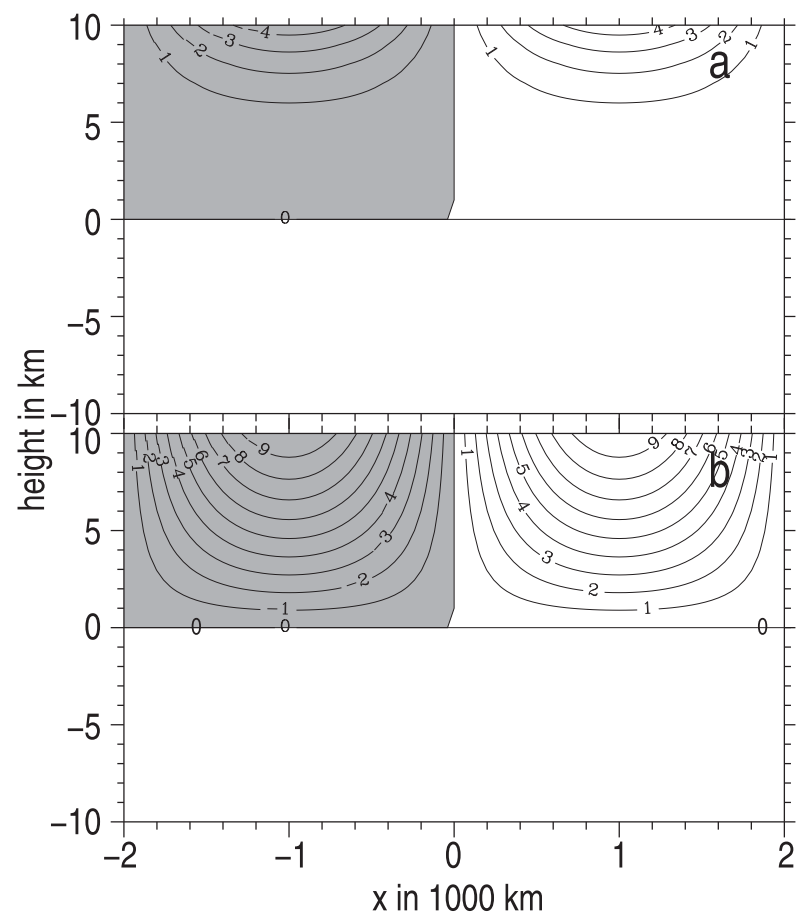

FIG. 4. (a) Streamfunction $\psi_{w}^{\prime}\left(10^{6} \mathrm{~m}^{2} \mathrm{~s}^{-1}\right)$ according to (14) and (b) $Q_{W}$ according to (15) in $10^{-5} \mathrm{~s}^{-1} ; N=10$.

$$
Q_{W}^{*}=2 P(3 z-H) \sin \left(k_{1} x\right)-k_{1}^{2} \psi_{w}^{*} .
$$

Both the streamfunction $\psi_{w}^{*}$ and $Q_{W}^{*}$ are displayed in Fig. 5 . There are two domains of $Q_{W}^{*}$ with different signs stacked above each other. There is no flow in D1.

\section{Discussion and conclusions}

The flow situation in the thought experiment of HMR has been analyzed here in order to settle questions concerning the far-field impact of PV anomalies and the role of boundary conditions in PPVI. The anomaly $Q_{L}$ is prescribed in quiescent mean flow above a layer of constant mean shear flow. This arrangement led HMR to conjecture that the flow obtained by inversion is stationary. Indeed, a stationary solution (10) has been found but without any "response" in the lower layer. This is a surprising result at least if a far-field influence of PV is expected. PDI has been conducted in parallel also without farfield response in D1.

The boundary conditions in the instationary cases in Fig. 3 are those accepted in standard PPVI. Cyclonic vorticity is found in D1 underneath positive PV anomalies as expected. The results of PPVI and PDI agree in the upper layer almost by definition. They should agree also in the lower layer if the anomalies in D2 exert an influence. However, PDI yields results which differ from those of PPVI in D1. This result led us to show that a physical downward impact of PV in D2 is impossible. Such an impact can, however, be ascribed to the density anomalies in the upper layer. This conclusion does, of course, not imply that PPVI is not useful. This procedure solves a

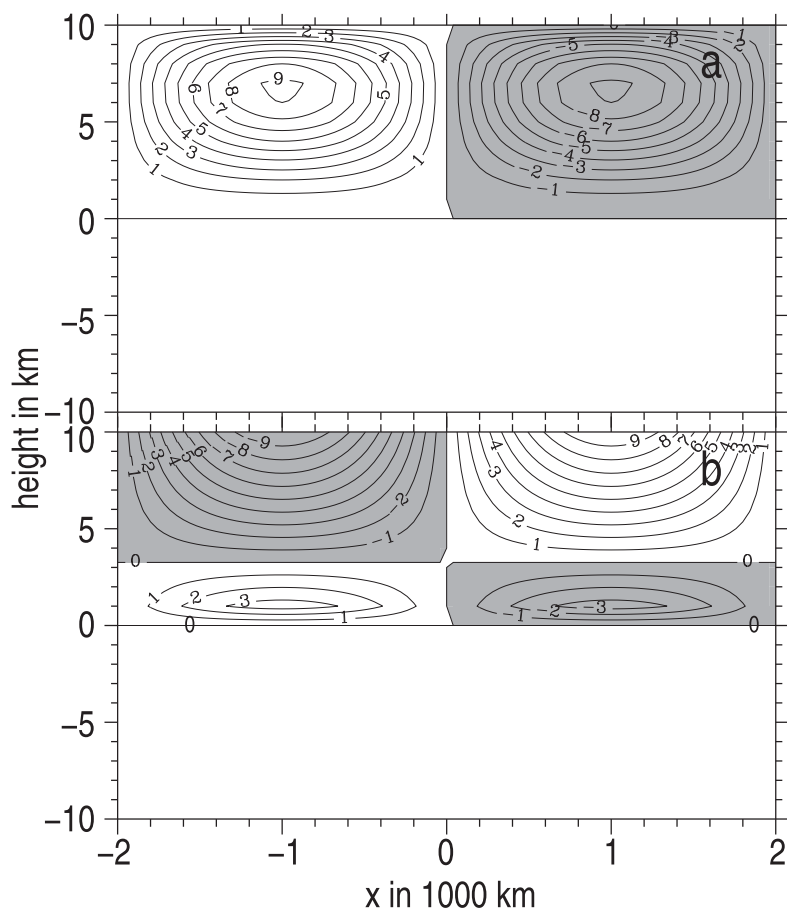

FIG. 5. As in Fig. 4, but for (a) $\psi_{w}^{\prime}$ according to (16) in $10^{5} \mathrm{~m}^{2} \mathrm{~s}^{-1}$ and (b) $Q_{W}(17)$ in $10^{-5} \mathrm{~s}^{-1}$.

mathematical problem and its solution tells us which flows form the inverted PV anomaly. A similar point has been made recently by McIntyre (2014), who stated that the "the term induced velocity means the velocity deduced from PV by inversion." The situation is even simpler with respect to the examples in section $3 \mathrm{~b}$ where $\mathrm{PV}$ is derived from $\psi$ by differentiation.

Several points need to be discussed, however. Strictly speaking "shielding" by PV anomalies is impossible because they do not exert an influence. Nevertheless it is helpful in the flow interpretation to insert PV anomalies formed by flows cancelling available "observed" flows. The wavy anomaly $Q_{W}$ is derived from a streamfunction which vanishes also in D1. Thus $Q_{W}$ is confined to $\mathrm{D} 2$ and is stationary. This approach excludes a far-field due to $Q_{W}$.

A fairly specific mean flow with a jump of mean temperature at $z=0$ is prescribed in the thought experiment of HMR. This flow has been chosen because stationary solutions can be found easily. However, neither PPVI nor PDI involve the profile of the mean flow. The results of inversions presented above are valid no matter which profiles of $U$ are chosen.

PDI can be applied in vertical direction only while the inversion of $q$ is circular symmetric. It is, however, difficult to conceive a lateral impact of PV if there is no vertical one. For example, one may define a streamfunction confined to a vertical strip with $\psi=0$ outside. The related $q$ is also confined.

The tests relied on the simplest formulation of PV. A more realistic height dependence of mean density would have complicated the solutions but would not have provided new insights with respect to induction. Inversions of more realistic 
forms of PV as in Davis (1992) are considerably more complicated than those of $q$. If, however, a far-field influence of PV would be found, this result would have to be valid in the quasigeostrophic case as well because the latter is a limiting case of general dynamics.

The tests have been conducted for a two-dimensional atmosphere. The step to those dimensions is simple and needs not to be discussed here.

Acknowledgments. Both referees invested much time and contributed significantly to the improvement of the manuscript. We appreciated particularly the open discussion with Dr. Heifetz during the review process.

\section{REFERENCES}

Baumgart, M., M. Riemer, V. Wirth, T. Teubler, and S. Lang, 2018: Potential vorticity dynamics of forecast errors. Mon. Wea. Rev., 146, 1405-1425, https://doi.org/10.1175/MWR-D-170196.1.

Bishop, C., and A. Thorpe, 1994: Potential vorticity and electrostatics analogy: Quasigeostrophic theory. Quart. J. Roy. Meteor. Soc., 120, 713-731, https://doi.org/10.1002/qj.49712051710.

Bleck, R., and C. Mattocks, 1984: A preliminary analysis of the role of potential vorticity in Alpine lee cyclogenesis. Beitr. Phys. Atmos., 57, 357-368.

Bretherton, F., 1966: Baroclinic instability and the short wavelength cutoff in terms of potential vorticity. Quart. J. Roy. Meteor. Soc., 92, 335-345, https://doi.org/10.1002/qj.49709239303.

Davies, H., and C. Bishop, 1994: Eady edge waves and rapid development. J. Atmos. Sci., 51, 1930-1946, https://doi.org/ 10.1175/1520-0469(1994)051<1930:EEWARD>2.0.CO;2.

Davis, C., 1992: Piecewise potential vorticity inversion. J. Atmos. Sci., 49, 1397-1411, https://doi.org/10.1175/1520-0469(1992) 049<1397:PPVI > 2.0.CO;2.
Egger, J., 2008: Piecewise potential vorticity inversion: Elementary tests. J. Atmos. Sci., 65, 2015-2024, https://doi.org/10.1175/ 2007JAS2564.1.

_ - and K.-P. Hoinka, 2010: Potential temperature and potential vorticity inversion complementary approaches. J. Atmos. Sci., 67, 4001-4016, https://doi.org/10.1175/2010JAS3532.1.

Hakim, G., D. Keyser, and L. Bosart, 1996: The Ohio valley wavemerger cyclogenesis event of 25-26 January 1978. Part II: Diagnosis using quasigeostrophic potential vorticity inversion. Mon. Wea. Rev., 124, 2176-2205, https://doi.org/10.1175/15200493(1996) $124<2176$ :TOVWMC $>2.0$.CO;2.

Hartley, D., J. Villarin, R. Black, and C. Davies, 1998: A new perspective of the dynamical link between the stratosphere and troposphere. Nature, 391, 471-474, https://doi.org/10.1038/ 35112.

Hoskins, B., M. McIntyre, and A. Robertson, 1985: On the use and significance of isentropic potential vorticity maps. Quart. J. Roy. Meteor. Soc., 111, 877-946, https://doi.org/10.1002/ qj.49711147002.

- M. Pedder, and D. Wynjones, 2003: The omega equation and potential vorticity. Quart. J. Roy. Meteor. Soc., 129, 32773303, https://doi.org/10.1256/qj.02.135.

McIntyre, M. E., 2014: Potential vorticity. Encyclopedia of Atmospheric Science, G. R. North, J. Pyle, and F. Zhang, Eds., Elsevier, 375-383.

Phillips, N., 1954: Energy transformation and meridional circulations associated with simple baroclinic waves in a two-level quasigeostrophic model. Tellus, 3A, 273-286, https://doi.org/ 10.1111/j.2153-3490.1954.tb01123.x.

Robinson, W., 1988: Analysis of LIMS data by potential vorticity inversion. J. Atmos. Sci., 45, 2319-2342, https://doi.org/ 10.1175/1520-0469(1988)045<2319:AOLDBP>2.0.CO;2.

Thorpe, A., 1997: Attribution and its application to mesoscale structure associated with tropopause folds. Quart. J. Roy. Meteor. Soc., 123, 2377-2399, https://doi.org/10.1002/qj. 49712354411. 